\title{
Experimental Tests of Methods for the Measurement of Rainfall Rate Using an Airborne Dual-Wavelength Radar
}

\author{
R. MENEGHINI \\ Goddard Space Flight Center, Greenbelt, Maryland \\ K. NAKAMURA \\ Radio Research Laboratory, Tokyo, Japan \\ C. W. ULBRICH \\ Clemson University, Clemson, South Carolina \\ D. ATLAS* \\ 7420 Westlake Terrace, Bethesda, Maryland
}

(Manuscript received 16 June 1988, in final form 27 December 1988)

\begin{abstract}
For a spaceborne meteorological radar, the use of frequencies above $10 \mathrm{GHz}$ may be necessary to attain sufficient spatial resolution. As the frequency increases, however, attenuation by rain becomes significant. To extend the range of rain rates that can be accurately estimated, methods other than the conventional $Z-R$, or backscattering method, are needed. In this paper, tests are made of two attenuation-based methods using data from a dual-wavelength airborne radar operating at $3 \mathrm{~cm}$ and $0.87 \mathrm{~cm}$. For the conventional dual-wavelength method, the differential attenuation is estimated from the relative decrease in the signal level with range. For the surface reference method, the attenuation is determined from the difference of surface return powers measured in the absence and the presence of rain. For purposes of comparison, and as an indication of the relative accuracies of the techniques, the backscattering, $(Z-R)$, method, as applied to the $3 \mathrm{~cm}$ data, is employed. As the primary sources of error for the $Z-R$, dual-wavelength, and surface reference methods are nearly independent, some confidence in the results is warranted when these methods yield similar rain rates. Cases of good agreement occur most often in stratiform rain for rain rates between a few $\mathrm{mm} \mathrm{h}^{-1}$ to about $15 \mathrm{~mm} \mathrm{~h}^{-1}$; that is, where attenuation at the shorter wavelength is significant but not so severe as to result in a loss of signal. When the estimates disagree, it is sometimes possible to identify the likely error source by an examination of the return power profiles and a knowledge of the error sources.
\end{abstract}

\section{Introduction}

An airborne rain-measuring experiment was conducted jointly by the Radio Research Laboratories (RRL) and NASA for the purpose of testing methods of rain estimation from space. The instrument was developed by RRL and installed in the NASA-P3 aircraft at Wallops Flight Facility, Wallops, Virginia. A series of flights were made in the autumn of 1985 and in June and July of the following year.

The RRL instrument consists of a dual-wavelength radar and radiometer. In the normal mode of opera-

\footnotetext{
* Consultant.
}

Corresponding author address: Mr. Robert Meneghini, NASA/ Goddard Space Flight Center, Code 675, Greenbelt, MD 20771. tion, the offset parabolic antennas are scanned crosstrack $\pm 23^{\circ}$ of nadir. The half-period, from one nadir observation to the next, is about $4 \mathrm{~s}$, during which time the aircraft, at a nominal speed of $200 \mathrm{knots}$ and an altitude of $5 \mathrm{~km}$, travels somewhat more than one-half the instantaneous field of view (IFOV). For about half the data that are presented in this paper, however, the antennas were fixed at nadir. As a single observation consists of a 128 sample average at both wavelengths, and as the pulse repetition frequency (PRF) is $440 \mathrm{~Hz}$, the averaged backscattered powers are available about every $1 / 3 \mathrm{~s}$, during which time the aircraft moves about $1 / 20$ of an IFOV. Some of the characteristics of the radar are shown in Table 1. A detailed description of the instrument and its operation is given by Okamoto et al. (1982). Below, we focus on the features of the instrument that make it suitable for testing rain-retrieval methods for spaceborne meteorological radars. 
TABLE 1. Characteristics of RRL airborne radar.

\begin{tabular}{lll}
\hline & $X$-Band & $K_{a}$-Band \\
\hline Frequency & $10 \mathrm{GHz}$ & $34.45 \mathrm{GHz}$ \\
Pealk power (Magnetron) & $20 \mathrm{~kW}$ & $10 \mathrm{~kW}$ \\
Pulse rep. freq. & $440 \mathrm{~Hz}$ & $440 \mathrm{~Hz}$ \\
Pulse duration & $0.5 \mu \mathrm{S}$ & $0.5 \mu \mathrm{s}$ \\
Receiver bandwidth & $2 \mathrm{MHz}$ & $2 \mathrm{MHz}$ \\
Receiver noise figure & $5.3 \mathrm{~dB}$ & $9.6 \mathrm{~dB}$ \\
Dynamic range & $80 \mathrm{~dB}$ & $80 \mathrm{~dB}$ \\
Antenna (Offset paraboloid) & & \\
$\quad$ Diameter & $260 \mathrm{~mm}$ & $75 \mathrm{~mm}$ \\
Gain & $26 \mathrm{~dB}$ & $26 \mathrm{~dB}$ \\
3 dB beamwidth & $8^{\circ} \times 8^{\circ}$ & $8^{\circ} \times 8^{\circ}$ \\
\hline
\end{tabular}

The frequencies of $10 \mathrm{GHz}$ ( $X$-band) and $35 \mathrm{GHz}$ ( $K_{a}$-band) span the range of frequencies that recently have been considered for the radar sensing of rain from space. For incidence angles within $\pm 20^{\circ}$ of nadir, the path length through the rain is typically less than 10 $\mathrm{km}$. For light to moderate rain rates, the $X$-band frequency can be considered to be nonattenuating. As such, the standard backscattering, or $Z-R$ method (where $Z$ is the equivalent radar reflectivity factor) can be employed. Despite drawbacks, both the simplicity and large dynamic range of the method make it indispensable for spaceborne rain measurements.

For frequencies much lower than $10 \mathrm{GHz}$, several difficulties arise. To achieve the same resolution at frequencies $f_{L}$ and $f_{H}$, the antenna area at the lower frequency, $f_{L}$, must be $\left(f_{H} / f_{L}\right)^{2}$ larger than that of the higher frequency, $f_{H}$. On the other hand, if the physical dimensions of the antenna and the available power at the antenna are held constant, the backscattered power from light rain at the higher frequency will increase by the ratio $\left(f_{H} / f_{L}\right)^{4}$. Furthermore, as the surface return is a relatively weak function of frequency, the use of a higher frequency tends to increase the ratio of the rain signal to surface clutter.

The addition of a higher frequency radar provides a possible way to extend the range of rain rates that can be estimated and may serve as a useful diagnostic tool for discrimination between the phase states of the hydrometeors. Although in this paper, the high and low radar frequency data are associated with the attenuation and backscattering methods, respectively, this linkage is not always necessary or desirable. For exarnple, use of the $Z-R$ method at the higher frequency may be preferable at light rain rates because of improved signal-to-noise ratios and reduced sensitivity to changes in the drop-size distribution (DSD). Conversely, for intense rain rates, where the rain and surface returns at the higher frequency are below the noise level, a single-wavelength attenuation method (such as the surface reference method) may be applicable at the lower frequency.

For the dual-wavelength method, the sensing of a common precipitation volume by both wavelengths may be critical to the accuracy (Rinehart and Tuttle 1982 ). For the RRL instrument, the $X$-band and $K_{a}$ band $3 \mathrm{~dB}$ beamwidths are approximately matched at $8^{\circ}$ with first side-lobe levels about $23 \mathrm{~dB}$ below the maximum on-axis gain. The transmitted pulses of 0.5 $\mu \mathrm{s}$ ( $75 \mathrm{~m}$ range resolution) are interleaved so that a common volume is viewed nearly simultaneously at the two frequencies.

A final point worth noting is that the dynamic range of the radar, $80 \mathrm{~dB}$, is usually sufficient to estimate both the rain and the surface returns. A dynamic range of this order is necessary for applying the surface reference methods.

\section{Description of methods}

In Fig. 1a and 1b, examples are shown of the rangecorrected radar return power at $10 \mathrm{GHz}(3 \mathrm{~cm}$ wavelength, solid line $)$ and $34.45 \mathrm{GHz}(0.87 \mathrm{~cm}$ wavelength, dashed line). Each return represents the average of 128 return waveforms. For both examples, the scale along the abscissa represents the range (in $\mathrm{km}$ ) as measured from the aircraft while the ordinate is $10 \log \left(P r^{2}\right)$ where $P$ is the return power in milliwatts and $r$ is the range in meters.

The data displayed in Fig. 1a were measured on 20 June 1986 over an ocean background for near-nadir incidence at an altitude of approximately $4.7 \mathrm{~km}$. This example shows stratification of the storm with a melting layer (bright band) at $X$-band of approximately $0.6 \mathrm{~km}$ depth beginning a distance of about $1.5 \mathrm{~km}$ below the aircraft. From the shape of the curves and the location of the melting layer we can infer a fairly uniform rainfall rate from $2.1 \mathrm{~km}$ to $4.5 \mathrm{~km}$ and a rapid increase in snow particle size or density between $0.5 \mathrm{~km}$ to 1.5 $\mathrm{km}$. At approximately $4.7 \mathrm{~km}$ a sharp rise in the signal levels indicates the return from the ocean surface. This is followed by a more gradual decrease in the return corresponding to the surface contributions from sidelobes progressively farther from the main lobe.

At $X$-band, a third component of the return is evident between $5.2 \mathrm{~km}$ and the maximum range of 9 $\mathrm{km}$. This mirror-image contribution appears to be caused by scattering of the incident pulse from the surface out to the rain, a portion of which is returned to the surface where it undergoes a secondary scattering from the surface back to the radar (Meneghini and Atlas 1986). In these examples, the $K_{a}$-band mirrorimage return beyond $6.5 \mathrm{~km}$ is obscured by the system noise.

Although a number of methods have been proposed for rain-retrieval from space, we will discuss only three: the standard $Z-R$, the dual-wavelength, and the surface reference methods. For the $Z-R$ or backscattering method the notation $Z_{X}-R, Z_{K}-R$ is used to distinguish the method as applied to the $X$-band or the $K_{a}$-band data, respectively. Dual-wavelength methods are categorized according to whether a range-averaging pro- 

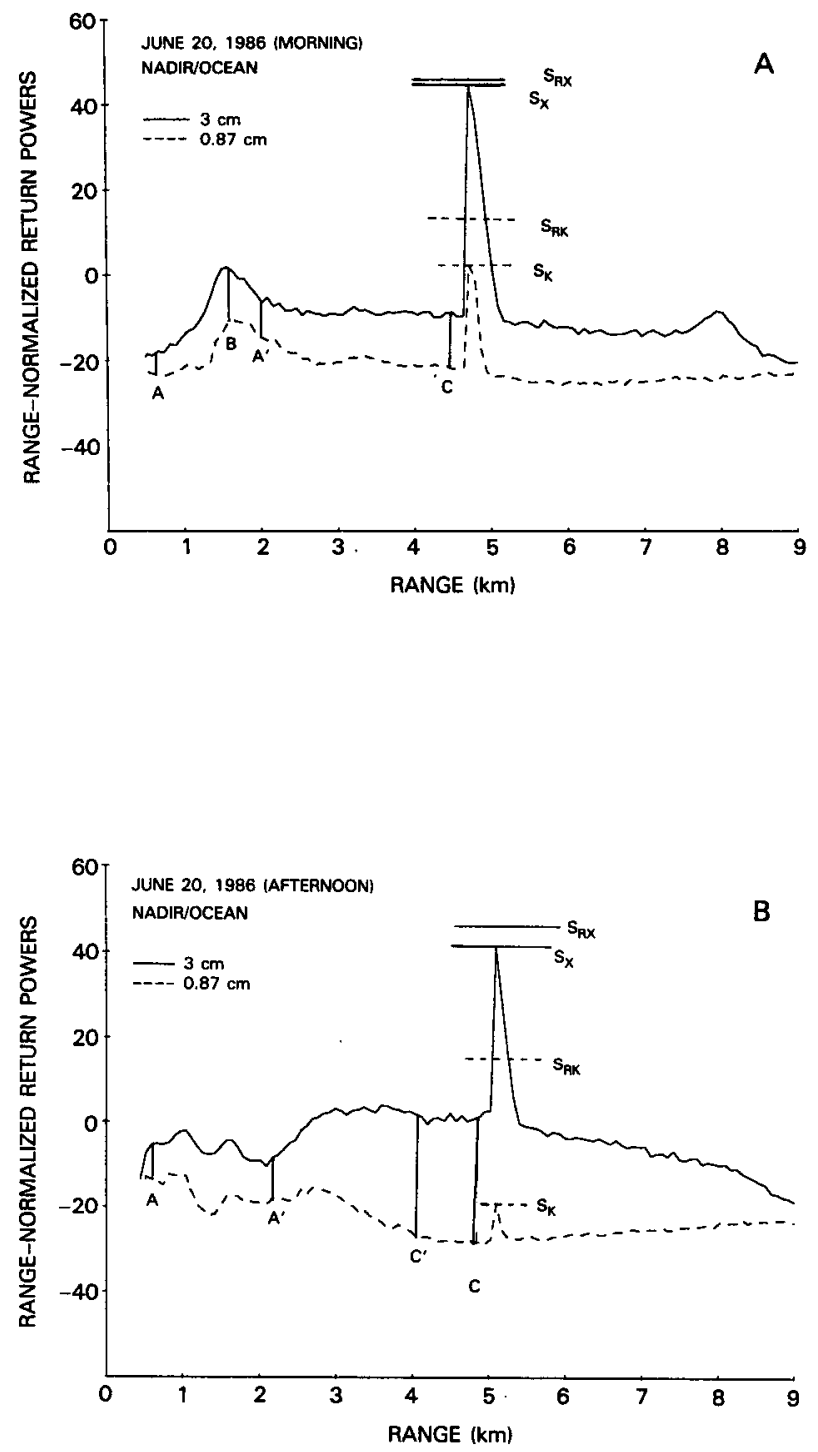

FIG. 1. Range-normalized return powers versus distance from the aircraft for $10 \mathrm{GHz}$ (solid line) and $34.45 \mathrm{GHz}$ (dashed line) frequencies for stratiform $(A)$ and convective $(B)$ rain. The variables $S_{R X}, S_{R K}$ denote the range-normalized return power from the surface at $X$-band and $K_{a}$-band, respectively, at an adjacent rain-free location while $S_{X}, S_{K}$ denote the corresponding surface returns with rain present. See text for further details.

cedure is used (DWAV) or whether the method is applied once over the entire interval (DWT). For the surface reference methods, both single wavelength (SRT) and dual-wavelength (DSRT) versions will be discussed.

\section{a. Radar reflectivity-rain rate (Z-R) method}

The curves of Fig. 1 can be used to illustrate the basic principle of each method. The most straightforward is the $Z-R$ method, where the equivalent radar reflectivity factor, $Z$, as found from the meteorological radar equation, requires estimates of the return power from the rain, the range, and the radar constant. By means of a reflectivity factor-rain rate relationship ( $Z$ $R$ ), the estimated $Z$ at each range gate is converted to $R$. Summing the rain rates at each range gate and dividing by the number of gates yields an estimate of the path-averaged rain rate.

Problems arise, however, as soon as this procedure is applied to the data in Fig. 1a. The simplest way to apply the method is to select an interval beginning near the storm top (point $\mathrm{A}$ ) and ending just above the surface (point $\mathrm{C}$ ). The interval $A C$, however, includes snow, rain and partially melted hydrometeors. If the $Z-R$ relationship for rain is employed throughout, significant errors can result. Although $Z-R$ relationships could be used that change according to the anticipated phase states and drop-size distributions within each level, such a procedure is not only laborious but depends a number of unknown parameters for the brightband and snow. A simpler alternative is to detect the bright-band and define the raining region to be some distance below. For example, in the interval $A^{\prime}$ to $C$, the probability is high that the hydrometeors are rain. The $Z-R$ relationships that will be used for the $X$-band and $K_{a}$-band returns in rain are:

$$
\begin{aligned}
& Z_{X}=300 R^{1.5} \\
& Z_{K}=355 R^{1.26} .
\end{aligned}
$$

The $Z_{K}-R$ and $k-R$ relationships in this paper are derived from the disdrometer measurements of Joss, as described by Atlas and Ulbrich (1977). Although a number of $Z_{X}-R$ laws were tested and varied in accordance with rain type, to simplify comparisons among the methods, a single "standard" $Z_{X}-R$ relationship was chosen.

\section{b. Dual-wavelength methods}

The basic idea of the dual-wavelength method is that the progressive divergence between the radar returns at two wavelengths is an indicator of the cumulative differential path-attenuation (Atlas 1954; Kostarev and Chernikov 1968; Eccles and Mueller 1971; Berjulev and Kostarev 1974). For example, by forming the difference between the radar returns at point $\mathrm{C}$ in Fig. 1a: $P(C)-P(C)$, and at point $\mathrm{A}: P(A)-P(A)$, where the powers are expressed in $\mathrm{dB}$, the differential attenuation in the interval $A C$ is found by a difference of differences:

$$
\begin{aligned}
{\left[P_{X}(C)-P_{K}(C)\right]-\left[P_{X}(A)-P_{K}(A)\right] } \\
\quad=2 \int_{A}^{C}\left[k\left(\lambda_{K}, r\right)-k\left(\lambda_{X}, r\right)\right] d r,
\end{aligned}
$$

where $k\left(\lambda_{X}\right), k\left(\lambda_{K}\right)$ are the specific attenuations (dB/ $\mathrm{km}$ ) at $3 \mathrm{~cm}$ and $0.87 \mathrm{~cm}$, respectively. Although in general, $k$ is given by the sum of contributions from 
atmospheric gases, cloud liquid water, and rain, we assume that the contributions from gases and cloud can be neglected.

The fundamental assumption of the dual-wavelength method is that the reflectivity factors at the endpoints of the interval are wavelength independent. For the interval $A C$ in Fig. 1a, this requires that $Z\left(\lambda_{X}\right)=Z\left(\lambda_{K}\right)$ at points $A$ and $C$. Even if this assumption were valid, converting the attenuation into rain rate would require $k-R$ relationships for various ranges within the snow and melting level. As with the backscattering method, a more straightforward procedure is to find a point $\mathrm{A}^{\prime}$ below the melting level and apply the method to the interval $A^{\prime} C$.

To relate the specific attenuation to rain rate, we use the disdrometer data of Joss (Atlas and Ulbrich 1977) which yield

$$
k\left(\lambda_{K}\right)-k\left(\lambda_{X}\right)=\alpha R^{\beta}=0.205 R^{1.04} .
$$

For the single wavelength surface reference method, the power law $k\left(\lambda_{K}\right)=\alpha^{\prime} R^{\beta^{\prime}}$ is used where $\alpha^{\prime}=0.22$ and $\beta^{\prime}=1.04$.

If we let the interval-averaged rain rate be approximated by

$$
\hat{R}=\frac{1}{r}\left[\int_{A^{\prime}}^{\grave{C}} R^{\beta}(r) d r\right]^{1 / \beta},
$$

where $r$ is the distance from $A^{\prime}$ to $C$, then from (2) and (3) $\hat{R}$ can be expressed in terms of measured quantities as

$$
\begin{aligned}
\hat{R}=1 / r\{(1 / 2 \alpha)[ & \left(P_{X}(C)-P_{K}(C)\right) \\
& \left.\left.-\left(P_{X}\left(A^{\prime}\right)-P_{K}\left(A^{\prime}\right)\right)\right]\right\}^{1 / \beta} .
\end{aligned}
$$

One additional feature, shown in Fig. $1 \mathrm{~b}$, must be considered in the selection of the interval. As in Fig. 1a, the beginning point of the interval $A$ is first shifted downward to $A^{\prime}$ to avoid what appears to be a region of non-Rayleigh scattering. Because the rain rate is more intense (and therefore the attenuation is greater) than in Fig. 1a, the $K_{a}$-band signal is masked by the system noise for ranges beyond about $4 \mathrm{~km}$. Because of this, the endpoint of the interval is shifted sufficiently far away from the surface (in this case from $C$ to $C^{\prime}$ ) so that the power level is indicative of the rain. The dual-wavelength method is then applied over the interval $A^{\prime} C^{\prime}$.

In the preceding discussion, the basic method is applied only once over the entire interval. Other methods have been proposed for range-averaging over the interval (Eccles and Mueller 1971; Eccles 1979; Meneghini 1977). To discuss one such method, let the beginning and end points of the interval be denoted by range bins 1 and $n$, respectively. For a single application of the method between bins 1 and $n$, we have from (2)

$$
\begin{aligned}
& \int_{r_{1}}^{r_{n}}\left[k\left(\lambda_{K}, r\right)-k\left(\lambda_{X}, r\right)\right] d r \\
& \quad=0.5\left\{\left[P_{X}(n)-P_{K}(n)\right]-\left[P_{X}(1)-P_{K}(1)\right]\right\}
\end{aligned}
$$

Letting

$$
\begin{aligned}
D(j, k)=0.5\left\{\left[P_{X}(j)-\right.\right. & \left.P_{K}(j)\right] \\
& \left.-\left[P_{X}(k)-P_{K}(k)\right]\right\}
\end{aligned}
$$

and

$$
A(j, k)=\int_{r_{k}}^{r_{j}}\left[k\left(\lambda_{K}, s\right)-k\left(\lambda_{X}, s\right)\right] d s,
$$

then (5) can be rewritten as

$$
A(n, 1)=D(n, 1) \text {. }
$$

The estimate of $A(n, 1)$ given by ( 8$)$ will be referred to as the standard dual-wavelength technique (DWT).

As an alternative estimate to $A(n, 1)$, consider a subinterval length $m(m<n)$ and application of the basic difference method $p$ consecutive times over the interval. This gives

$$
A^{\prime}(n, 1)=((n-1) / p m) \sum_{j=1}^{p} D(m+j, j),
$$

where $m+p=n$. For the case $m=n-1, p=1$, the estimates of interval attenuation given by (5) and (8) are identical. If we minimize that part of the variance of $A^{\prime}$ caused by finite sampling, we find that $p$ is to be chosen as the integer nearest $n / 3$ which leads to a subinterval length, $m$, equal to $n-\operatorname{int}(n / 3)$ (Meneghini 1977). The estimate given by (9) will be referred to as the dual-wavelength averaging method (DWAV). An example of the range bin selections that are implied by the above condition is shown in Fig. 2 for the case of $n=12, p=4, m=8$. The price paid in the attempt to reduce the variance of the estimate is an increase in the bias. This occurs because the range bins near the center of the interval are more highly weighted than those near the ends.

\section{c. Surface reference techniques}

The third method discussed in this paper is the surface reference (SR) technique. In the single wavelength version (SRT), the backscattered power from the surface measured in the absence of rain, $S_{R K}$, is compared to the surface return in the presence of rain, $S_{K}$. To the extent that the backscattering properties do not change appreciably between the measurements, the difference (in dB) between $S_{R K}$ and $S_{K}$ provides an estimate of the total path attenuation (Meneghini et al. 1983; Fujita et al. 1985):

$$
0.5\left(S_{R K}-S_{K}\right)=\int_{0}^{r} k\left(\lambda_{K}, r\right) d r=A_{\mathrm{SRT}}
$$


SUBINTERVAL

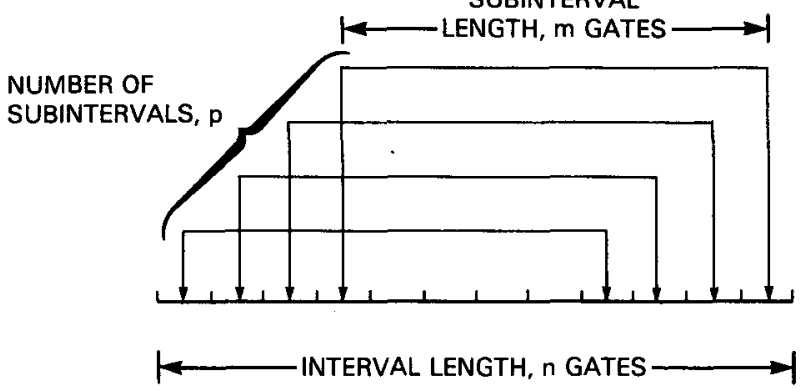

FIG. 2. Schematic of subinterval averaging for the dual-wavelength method for the case of $n=12, p=4$, and $m=8$, where $n, m$ are the number of range gates comprising the interval and subinterval, respectively, and $p$ is the number of subintervals.

where $r$ is the total path length through the hydrometeors.

The dual-wavelength version of the method (DSRT) is analogous to the DWT and involves a difference of differences at two wavelengths (Moore 1981; Meneghini et al. 1987):

$$
\begin{aligned}
& 0.5\left[\left(S_{R K}-S_{K}\right)-\left(S_{R X}-S_{X}\right)\right] \\
& \quad=\int_{0}^{r}\left[k\left(\lambda_{K}, r\right)-k\left(\lambda_{X}, r\right)\right] d r=A_{\mathrm{DSRT}} .
\end{aligned}
$$

In Fig. 1a and $1 \mathrm{~b}$, the range-normalized reference values $S_{R K}$ and $S_{R X}$, for $K_{a}$ - and $X$-band respectively, as well as the corresponding values measured within the rain, $S_{K}$ and $S_{X}$, are indicated by the horizontal lines. The reference values were found from measurements made at a nearby rain-free region.

The surface reference can be chosen in a number of ways. For example, an individual measurement of the surface return can be defined either as the maximum value or the average over all gates that intersect the surface. Moreover, the reference measurement can be defined either as the spatial average of individual measurements over the rain-free areas or as the temporal average at a particular location taken before or after the rain event. For most of the results given here, the surface reference for each incidence angle and background type (land/ocean) is chosen as the average over the entire flight of surface return data gathered in rainfree regions. (In some cases, the surface reference data were further categorized according to the aircraft altitude.) In all cases, an individual reference measurement is taken to be the maximum backscattered power from the surface where the maximum value is found by fitting a quadratic through the three largest values of the surface return and interpolating.

A final question is whether an account can be made for attenuation from sources other than rain. Unlike the $Z-R$ and dual-wavelength methods, the interval used in the surface reference methods is not adjustable. Consequently, either the entire column is treated as if it were rain or $k-R$ relationships are used that depend on the expected phase states and drop-size distributions at each level. For the first case, the path-averaged rain rate estimates for the SRT and DSRT, respectively, are

$$
\begin{gathered}
R_{\mathrm{SRT}}=\frac{1}{r}\left[\left(0.5 / \alpha^{\prime}\right)\left(S_{R K}-S_{K}\right)\right]^{1 / \beta^{\prime}} \\
R_{\mathrm{DSRT}}=\frac{1}{r}\left\{( 0 . 5 / \alpha ) \left[\left(S_{R K}-S_{K}\right)\right.\right. \\
\left.\left.-\left(S_{R X}-S_{X}\right)\right]\right\}^{1 / \beta} .
\end{gathered}
$$

In this paper, the second case is not considered.

\section{Results}

The objectives of this and the following sections are to present an overview of the experimental results and infer some general characteristics of the methods outlined above.

\section{a. Light rain rate cases}

To help organize the results, cases of light rainfall rates are discussed first. Throughout the paper, the rainfall estimates are presented either by a temporal or a scatter plot. In all cases, the results of the $Z_{X}-R$ method are shown for purposes of comparison.

The temporal plots in Figs. 3 and 4 show the pathaveraged rain rate estimates for five of the above methods as a function of observation number. The dotted curves in each of the figures represent the path-averaged rain rates as found from the $Z_{X}-R$ method. The results are derived from measurements taken on 9 July 1986 at near-nadir incidence. As noted earlier, a single observation is taken approximately every $1 / 3 \mathrm{~S}$ and consists of an average of 128 backscattered waveforms at both frequencies. Unfortunately, it is difficult to associate a distance scale with these plots as data was collected in both scanning and nonscanning modes. If we assume the data to be equally divided between the two modes, and note that the aircraft travels about $0.4 \mathrm{~km}$ between each nadir observation while in the scanning mode and about $0.034 \mathrm{~km}$ while in the nonscanning mode, then 1000 points represent a distance on the order of $220 \mathrm{~km}$.

In Fig. 3a, a comparison is shown between the $Z_{K^{-}}$ $R$ (solid line) and the $Z_{X}-R$ methods. For rain rates less than about $2 \mathrm{~mm} \mathrm{~h}^{-1}$, the correlation is excellent; above this level, because of attenuation effects, the $Z_{K^{-}}$ $R$ estimate rapidly saturates so that it appears as a clipped version of the $Z_{X}-R$ curve. Although attenuation correction algorithms can be applied to the $K_{a}$ band data, these will not be discussed in this paper.

To understand this fairly sharp cutoff in the $Z_{K}-R$ estimate, we note that on using a power law relating the specific attenuation $k$ and the equivalent reflectivity factor $Z\left(k=p Z^{q}\right)$, the radar equation can be written 

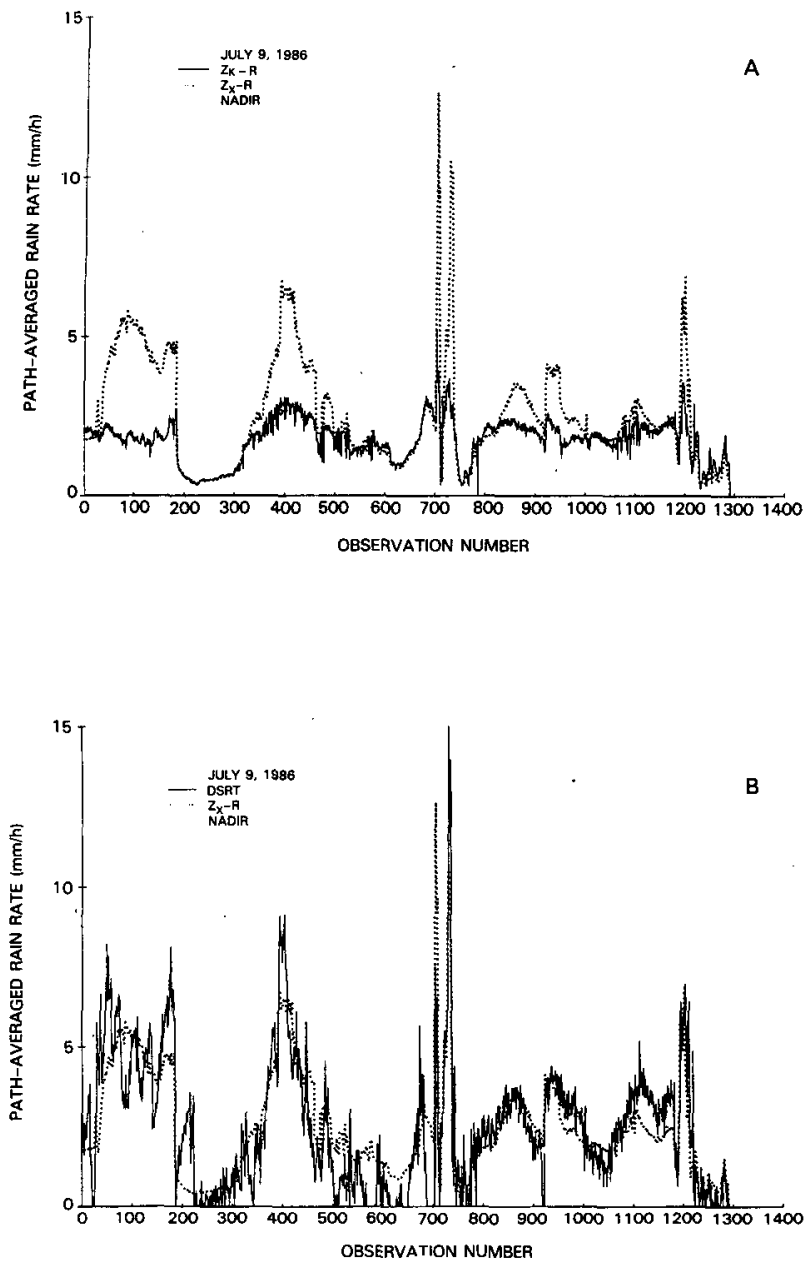

FIG. 3. (A) Path-averaged rain rates as a function of observation number for the $Z_{X}-R$ (dotted line) and $Z_{K}-R$ (solid line) methods for data measured on 9 July 1986. (B) Same as (A) but with the solid line representing the dual-wavelength surface reference technique (DSRT).

$$
r^{2} P / C=Z_{m}(r)=Z(r) \exp \left(-0.46 p \int_{0}^{r} Z^{q}(r) d r\right),
$$

where $Z_{m}$ is the measured or apparent radar reflectivity factor discounting attenuation. Using $R=a Z_{m}^{b}$, the path-averaged rain rate over the interval from 0 to $r_{n}$ is

$$
R=\frac{a}{r_{n}} \int_{0}^{r_{n}} Z_{m}^{b}(r) d r
$$

Assuming that $Z(r)=Z_{0}$, expressing $Z_{m}$ in terms of $Z_{0}$ and integrating gives

$$
R=a Z_{0}^{(b-q)}\left[1-\exp \left(-0.46 p b r_{n} Z_{0}^{q}\right)\right] / 0.46 p b r_{n} .
$$

The nearly linear relationship between $k$ and $R$ at $35 \mathrm{GHz}$ (Atlas and Ulbrich 1977) implies that $b$ and $q$ are approximately equal; in particular, from the dis- drometer measurements of Joss, $b=0.77, q=0.8$. Thus, as the total attenuation increases, the exponential term in the above equation tends to zero and $R$ approaches a value that is virtually independent of the reflectivity factor $Z_{0}$. This explains, at least qualitatively, the abrupt leveling off of the curve in Fig. 3a.

In Fig. 3b, results of the dual-wavelength surface reference technique (DSRT) are represented by the solid line. The curves in this case are useful in comparing the performance of the method over land and ocean backgrounds. Over the second half of the dataset (observation numbers greater than 700 ), where the background is ocean, the estimates from the $Z_{X}-R$ and DSRT methods closely track one another. The correlation coefficients over this span are 0.91 for the DSRT and $Z_{X}-R$ and 0.82 for the SRT and $Z_{X}-R$. Over land background (observation numbers less than 700 ), the correlation coefficients between the DSRT and $Z_{X}-R$
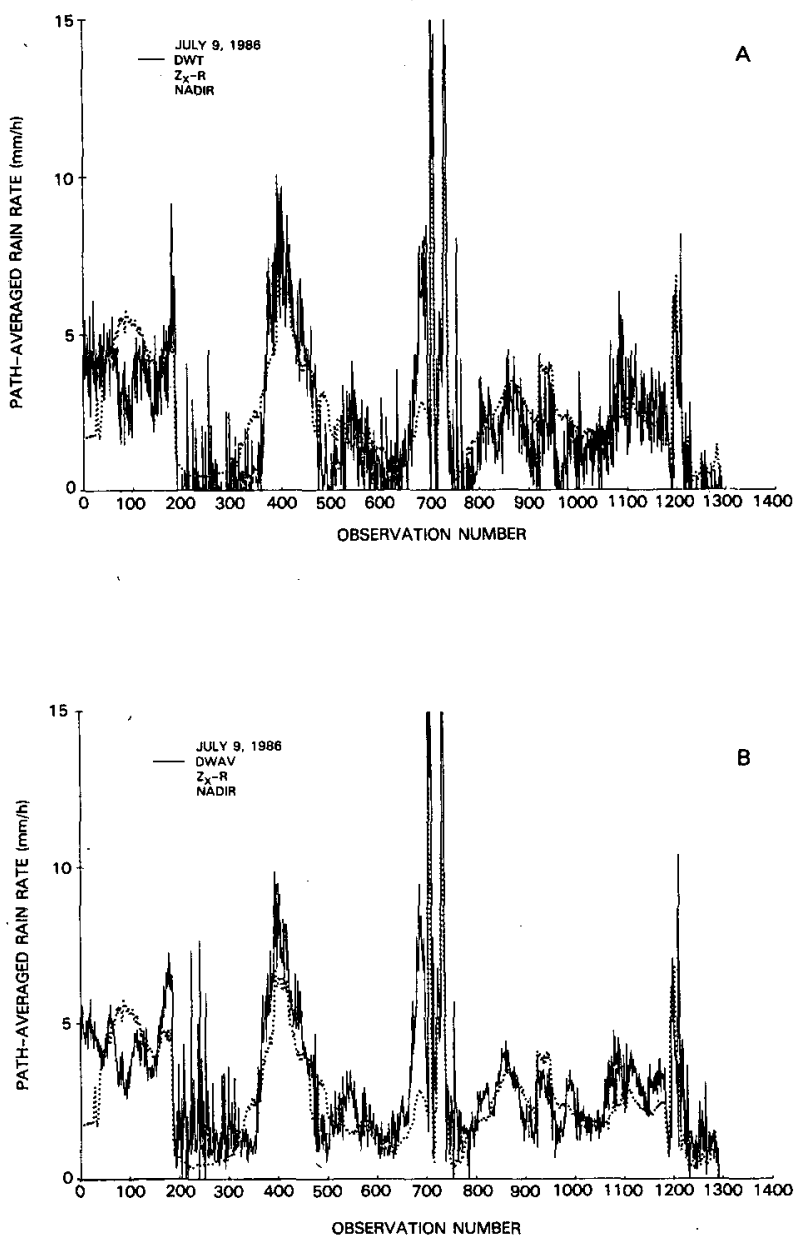

FiG. 4. (A) Path-averaged rain rates as a function of observation number for the $Z_{X}-R$ (dotted line) and the dual-wavelength (DWT) (dashed line) methods for data measured on 9 July 1986. (B) Same as (A) but with the solid line representing the dual-wavelength rangeaveraging (DWAV) method. 


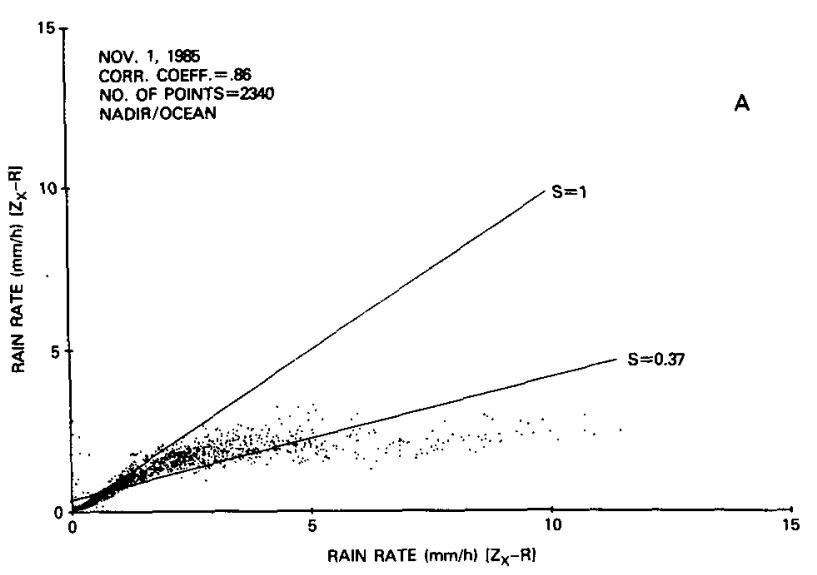

backscattering cross section, $\sigma^{\circ}$ (i.e., scattering cross section per unit area), over successive fields of view and a slower variation which may represent a change in the type of terrain.

In Fig. 4 the rain rates as derived from the dualwavelength methods are shown for the DWT (without range averaging, Fig. 4a) and for the DWAV (with range-averaging, Fig. 4b). As with the surface reference, the dual-wavelength methods exhibit fine-scale oscillations that almost certainly are unrelated to the actual rainfall rate. Use of the range-averaging method in this case generally lessens the variability except in instances of very light rain (e.g., observation numbers from 200 to 300 ).
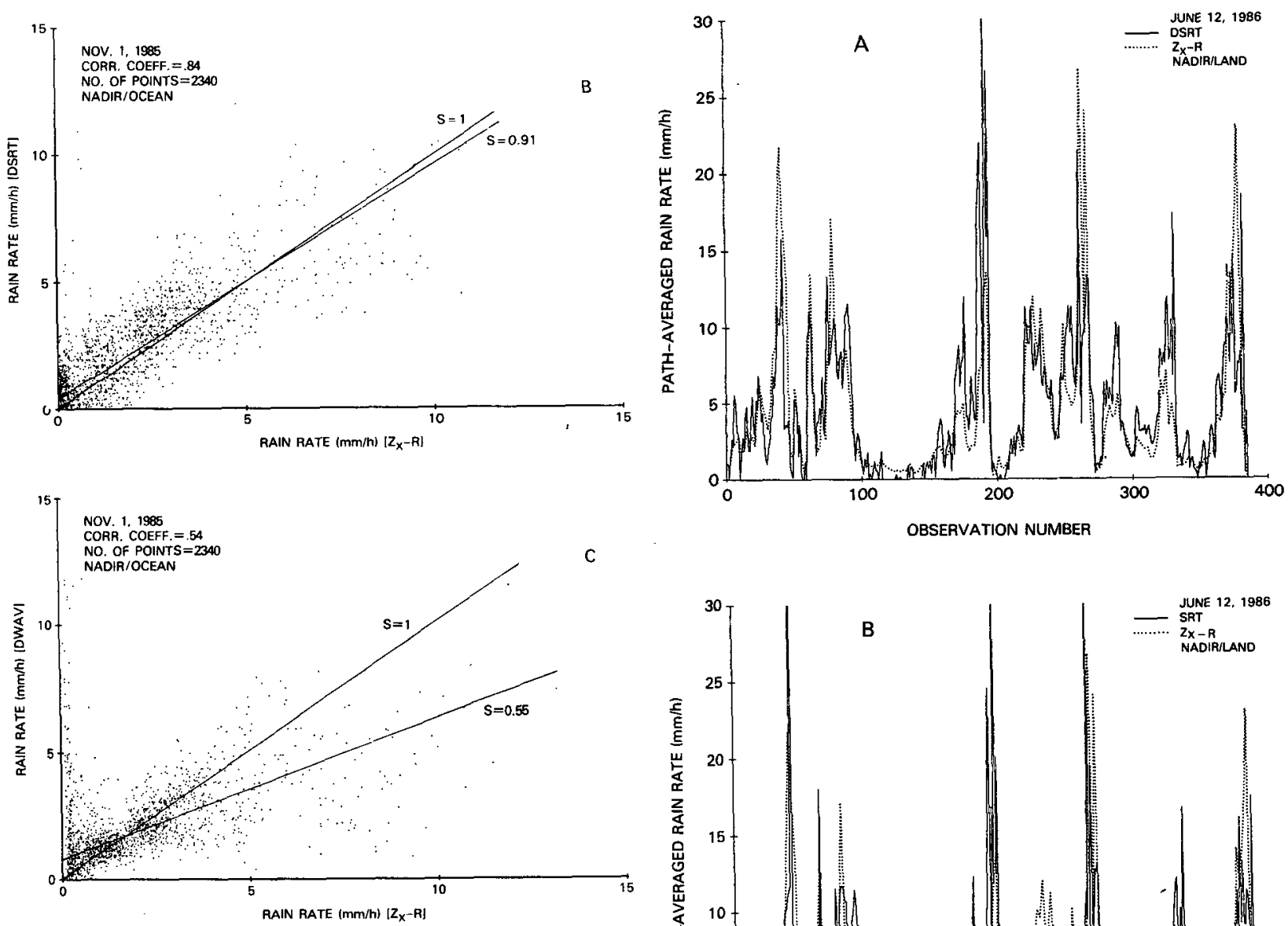

FIG. S. Scatter plots of path-averaged rain rates as estimated by the $Z_{X}-R$ method (abscissa) versus the $Z_{K}-R$ method (A), the DSRT (B) and the DWAV (C) for data measured over an ocean background on 1 Nov. 1985. The slopes, $S$, of the regression lines are shown for each case; lines of slope equal to 1 are shown for purposes of comparison.

and between the SRT and $Z_{X}-R$ decrease to 0.7 and 0.63 , respectively. The results of the surface reference over land suggest variations on two time scales: Rapid fluctuations caused by variations in the normalized

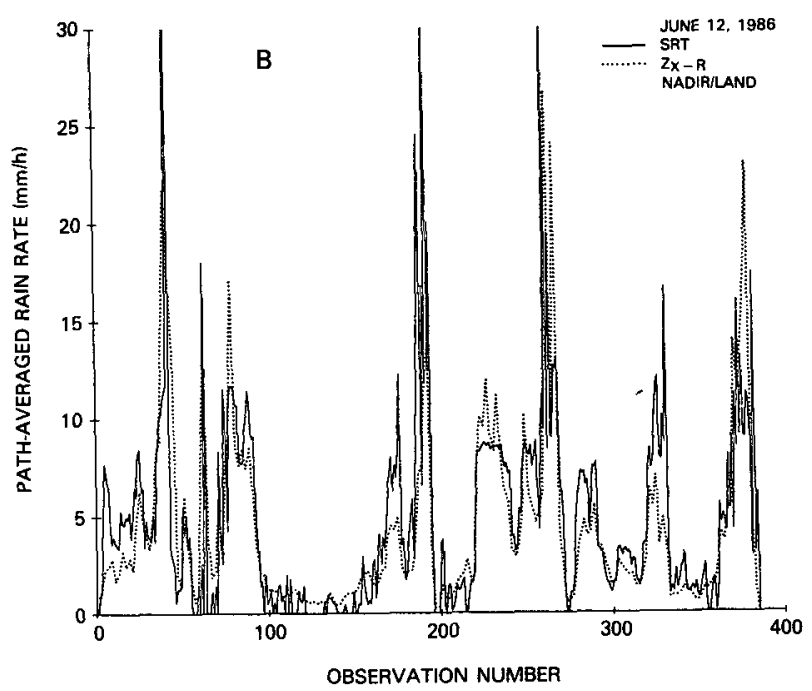

FIG. 6. (A) Path-averaged rain rates as a function of observation number for the $Z_{X}-R$ (dotted line) and the DSRT for data measured over land on 12 June 1986. (B) Same as (A) but with the solid line representing the single wavelength surface reference technique (SRT). 
As noted earlier, the interval over which the dualwavelength methods are applied is chosen to be below the melting level but far enough above the surface to ensure that the rain contribution dominates the surface scattering and the system noise. The procedure used to select the starting point of the interval is to locate the range beyond which the $3 \mathrm{~cm}$ and $0.87 \mathrm{~cm}$ returns diverge monotonically. Regions of nonliquid hydrometeors are defined to be above this gate and rain below. The selection of the end point is a three-step procedure. Having located the range gate at which the surface return is maximum, we move $m$ gates (where $m$ is a function of beamwidth and incidence angle) toward the radar to minimize contamination by surface scattering. At this gate, a test is made to determine whether the returns exceed the system noise. If not, the process is continued until a gate is reached where the signal levels are acceptably large. For the results shown in Figs. 4-8, $m$ is between 3 and 5 .

Of course, a simpler procedure would be to define the starting point as the gate nearest the radar for which the returns are above a certain threshold. The drawback, however, is that the point sometimes will be chosen within the bright-band, where the basic assumption of the method is invalid. Using this simple procedure for the 9 July dataset gives a correlation coefficient between the DWT and $Z_{X}-R$ methods of 0.54 , as compared to the above procedure which yields a value of 0.76 .

A summary of results for this flight is presented in Table 2. It should be noted that since the statistics have been computed for three near-nadir angles, the number of observations (1910) is larger than that in Figs. 3 and 4 where results from only a single incidence angle are shown. The mean and standard deviation of the rain rate estimates are given in the top portion of the table.

To explain the two sets of results in Table $2 \mathrm{a}$ we first note that the decision as to whether rain is present along the beam is determined by a threshold test: If the $X$-band and $K_{a}$-band returns exceed by some level their respective mean receiver noise powers for any three contiguous range gates, then rain is assumed to be present. The nature of the test ensures that the backscattering methods yield nonzero rain rates. For light rain, however, one or more of the attenuation methods occasionally yield negative rain rates which are then set to zero. In Table $2 \mathrm{a}$ and Table 3 the means and standard deviations have been computed both for the full dataset and for only those rain rates that exceed $0.01 \mathrm{~mm} \mathrm{~h}^{-1}$ (entries in parentheses). All other results in this paper include cases of zero rain rate.

By including all of the data, good agreement in the means is obtained for the DSRT, DWT, and $Z_{X}-R$ methods whereas the SRT and DWAV methods are approximately $0.6 \mathrm{~mm} \mathrm{~h}^{-1}$ larger. By eliminating cases of zero rain rate, the bias in the attenuation methods increases relative to the $Z_{X}-R$ result. As expected, the
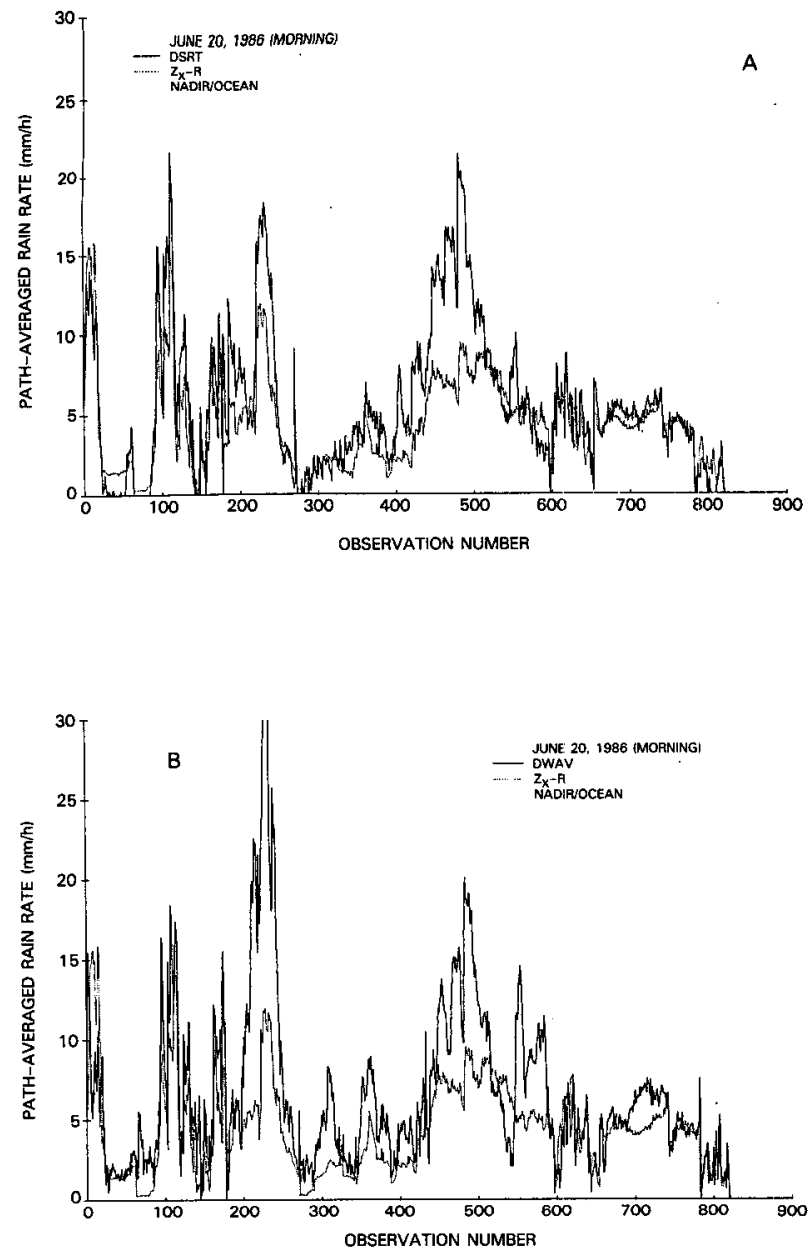

FIG. 7. (A) Path-averaged rain rates as a function of observation number for the $Z_{X}-R$ (dotted line) and the DSRT (solid line) for data measured over the ocean on 20 June 1986 (morning). (B) Same as $(A)$ but with the solid line representing the DWAV method.

rain rates estimates from the $Z_{K}-R$ method are negatively biased because of attenuation.

The matrix of correlation coefficients are listed in the second part of Table 2 . Correlation coefficients of $Z_{X}-R$ with the five other methods exhibit little variation, ranging from a low of 0.7 (SRT) to a high of $0.8\left(Z_{K}-R\right)$. It is worth noting that the correlation coefficient for the SRT and DSRT is only 0.62 , implying that the addition of a second wavelength can markedly affect the behavior of the surface reference method. On the other hand, the high correlation between the DWT and DWAV (0.95) indicates these methods respond similarly to the sources of error and to the rain rate.

In the bottom portion of the table are shown the normalized errors for the various combinations of methods. Using $R_{A_{j}}$ and $R_{B_{j}}$ for the rain rate estimates at the $j$ th observation for methods $A$ and $B$ respectively, the normalized error is defined to be the rms (root- 

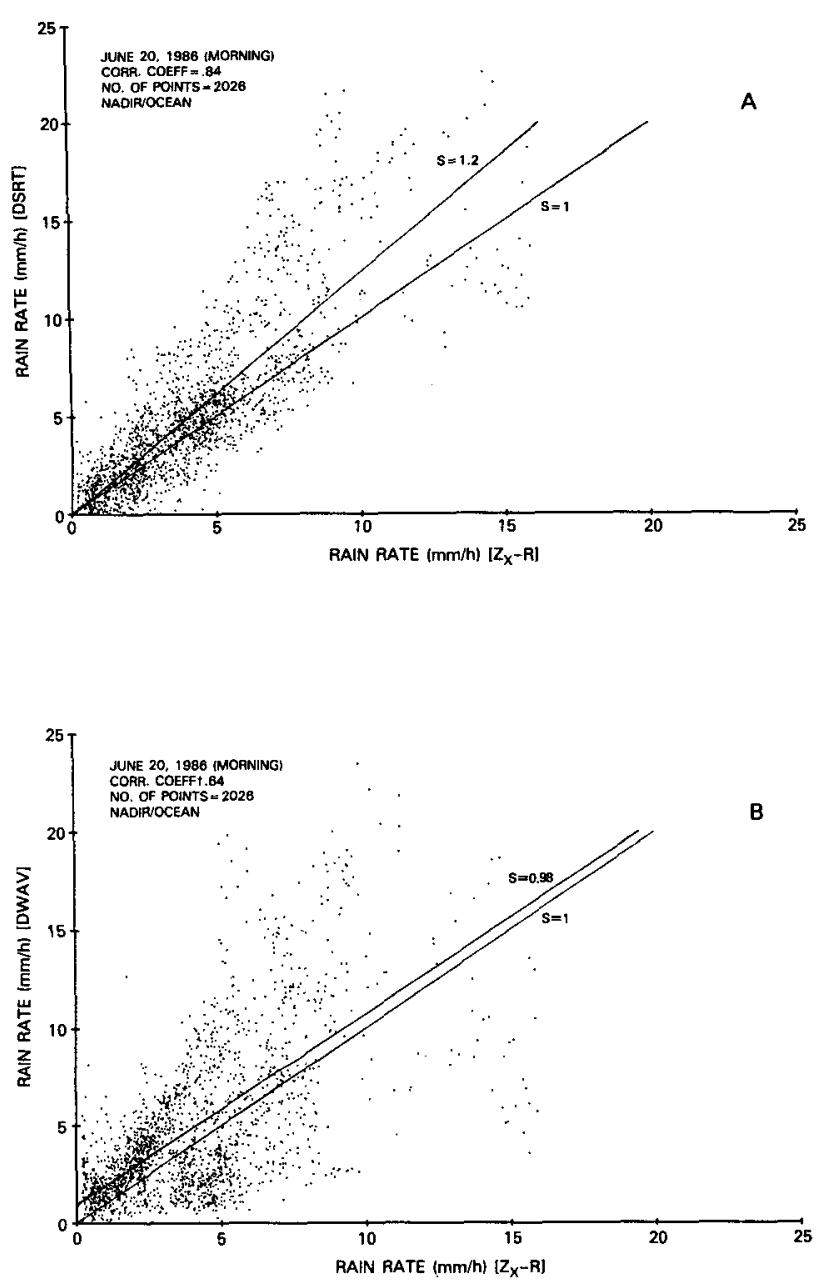

FIG. 8. Scatter plots of path-averaged rain rates as estimated by the $Z_{X}-R$ method (abscissa) versus the DSRT (A), and the DWAV method (B) for near-nadir incidence over ocean for data measured on 20 June 1986 (morning). The slopes, $S$, of the regression lines are shown for each case; lines of slope equal to 1 are shown for purposes of comparison.

mean-square) value of the differences divided by the average rain rate (which is defined as the average of the sample means, $\bar{R}_{A}, \bar{R}_{B}$ as computed from methods $A$ and $B$ ):

$$
N E=\frac{2\left[1 / N \sum_{j=1}^{N}\left(R_{A_{j}}-R_{B_{j}}\right)^{2}\right]^{1 / 2}}{\bar{R}_{A}+\bar{R}_{B}},
$$

where $N$ is the total number of observations (1910). Examination of the top row of the matrix shows that the method in closest agreement with the $Z_{X}-R$ is the DSRT (0.59) followed by the DWAV (0.72).

The scatter plots of Fig. 5 illustrate the path-averaged rain rates derived from the $Z_{K}-R$, DSRT, DWAV and $Z_{X}-R$ methods for measurements made on 1 Nov. 1985. The majority of data were collected over ocean
TABLE 2. Statistics for 9 July 1986 flight $(N=1910)$.

\begin{tabular}{lcc}
\hline \hline a. Mean and standard deviation* & \\
Method & $\begin{array}{c}\text { Mean } \\
\left(\mathrm{mm} \mathrm{h}^{-1}\right)\end{array}$ & $\begin{array}{c}\text { Std Dev } \\
\left(\mathrm{mm} \mathrm{h}^{-1}\right)\end{array}$ \\
\hline$Z_{X}-R$ & $2.6(2.6)$ & $1.7(1.7)$ \\
$Z_{K}-R$ & $1.7(1.7)$ & $0.75(0.75)$ \\
DSRT & $2.5(2.8)$ & $2.2(2.2)$ \\
SRT & $3.2(3.7)$ & $3.0(2.9)$ \\
DWT & $2.5(3.1)$ & $3.1(3.2)$ \\
DWAV & $3.3(3.3)$ & $3.1(3.1)$
\end{tabular}

b. Matrix of correlation coefficients

\begin{tabular}{lccccc} 
& $Z_{K}-R$ & DSRT & SRT & DWT & DWAV \\
\hline$Z_{X}-R$ & \multirow{2}{*}{0.80} & 0.76 & 0.70 & 0.76 & 0.77 \\
$Z_{K}-R$ & & 0.61 & 0.54 & 0.65 & 0.64 \\
DSRT & & & 0.62 & 0.55 & 0.54 \\
SRT & & & & 0.50 & 0.47 \\
DWT & & & & & 0.95
\end{tabular}

c. Normalized error

\begin{tabular}{llllll}
$Z_{X}-R$ & 0.81 & 0.59 & 0.75 & 0.80 & 0.72 \\
$Z_{X}-R$ & & 0.95 & 1.22 & 1.32 & 1.23 \\
DSRT & & & 0.88 & 1.07 & 0.97 \\
SRT & & & & 1.09 & 0.95 \\
DWT & & & & & 0.43 \\
\hline
\end{tabular}

* Entries not in parentheses include all data; entries in parentheses include only those data for which the rain rate exceeds $0.01 \mathrm{~mm} \mathrm{~h}^{-1}$.

and, for the results shown, the incidence angle is within $5^{\circ}$ of nadir. The top figure shows excellent agreement between the $Z-R$ methods for rain rates less than a few $\mathrm{mm} \mathrm{h}{ }^{-1}$ with a correlation coefficient for the entire dataset of 0.86 .

Although comparisons of the DSRT and $Z_{X}-R$ data in Fig. 5b show an increase in the amount of scatter relative to Fig. 5a, the correlation coefficient for this case $(0.84)$ is only slightly smaller than in Fig. 5a because of the improved correspondence at higher rain rates. Comparisons between the DWAV and the $Z_{X}-R$ (Fig. 5c) exhibit a decrease in the correlation to 0.54 . By eliminating cases for which either estimate is

TABLE 3. Mean and standard deviation of 1 Nov. 1985 flight $(N=2340)$.*

\begin{tabular}{lcc}
\hline \hline Method & $\begin{array}{c}\text { Mean } \\
\left(\mathrm{mm} \mathrm{h}^{-1}\right)\end{array}$ & $\begin{array}{c}\text { Std Dev } \\
\left(\mathrm{mm} \mathrm{h}^{-1}\right)\end{array}$ \\
\hline$Z_{X}-R$ & $1.5(1.5)$ & $1.8(1.8)$ \\
$Z_{K}-R$ & $0.9(0.9)$ & $0.8(0.8)$ \\
DSRT & $1.7(2.5)$ & $1.9(1.9)$ \\
SRT & $1.8(2.5)$ & $2.3(2.4)$ \\
DWT & $1.1(2.3)$ & $1.8(2.0)$ \\
DWAV & $1.5(2.2)$ & $1.8(1.8)$ \\
\hline
\end{tabular}

* Entries not in parentheses include all data; entries in parentheses include only those data for which the rain rate exceeds $0.01 \mathrm{~mm} \mathrm{~h}^{-1}$. 
less than $1 \mathrm{~mm} \mathrm{~h}^{-1}$, the correlation between the $Z_{X}-R$ and DWAV improves to 0.64 . The means and standard deviations of the estimates for this flight are given in Table 3. Despite generally low values in the correlation coefficient, the mean values are in fair agreement.

\section{b. Error sources}

Each of the methods described above is subject to a large number of errors. Discussion of some of the major error sources is given below.

For the surface reference methods a primary source of error arises from the fluctuations in the normalized scattering cross section of the surface, $\sigma^{\circ}$. Simplified expressions for the bias, $B$, and error variance of the SRT and DSRT are (square-law detection)

$$
\begin{aligned}
B_{\mathrm{SRT}} & =0.5\left[\tilde{E}\left(\lambda_{K}\right)-E\left(\lambda_{K}\right)\right] \\
\operatorname{var}\left(A_{\mathrm{SRT}}\right) & =\frac{4.71}{M}+0.25 s^{2}\left(\lambda_{K}\right)
\end{aligned}
$$

and

$$
B_{\mathrm{DSRT}}=0.5\left[\tilde{E}\left(\lambda_{K}\right)-E\left(\lambda_{K}\right)-\tilde{E}\left(\lambda_{X}\right)+E\left(\lambda_{X}\right)\right]
$$

$$
\begin{aligned}
\operatorname{var}\left(A_{\mathrm{DSRT}}\right)=\frac{9.43}{M} & +0.25\left[s^{2}\left(\lambda_{X}\right)+s^{2}\left(\lambda_{K}\right)\right. \\
& \left.-2 \rho\left(\lambda_{X}, \lambda_{K}\right) s\left(\lambda_{X}\right) s\left(\lambda_{K}\right)\right],
\end{aligned}
$$

where $M$ is the number of independent samples, $E\left(\lambda_{X}\right)$, $\tilde{E}\left(\lambda_{X}\right)$ are the mean values of $\sigma^{\circ}(\mathrm{dB})$ at $X$-band in raining and rain-free regions, respectively, $s^{2}\left(\lambda_{X}\right)$, $s^{2}\left(\lambda_{K}\right)$ are the variances of $\sigma^{\circ}$ at $X$-band and $K_{a}$-band, respectively, while $\rho\left(\lambda_{X}, \lambda_{K}\right)$ is the correlation coefficient of $\sigma^{\circ}$ at the two wavelengths. In (15) and (17) all quantities refer to the statistics of $\sigma^{\circ}$ within the rain.

Equations (15) and (17) differ in several respects from previous results (Meneghini et al. 1987). For the processing of the experimental data, the surface reference measurement is defined not as a single observation at a rain-free region, as was done previously, but as an average at a fixed incidence angle and background type over the nonraining area. As the variability in the averaged reference measurement is small relative to the fluctuations from observation to observation, the reference measurement is treated as a constant. Consequently, the correlation between $\sigma^{\circ}$ measured within and outside the rain is taken to be zero and the expressions for the error variances are multiplied by one-half. In addition, the following approximation, valid for large $M$, is used:

$$
\pi^{2} / 6-\sum_{j=1}^{M-2}\left(1 / j^{2}\right)=1 / M
$$

Using $M=128$ and replacing the statistics in (15) and (17) with the corresponding sampled statistics de- rived from data measured in rain-free areas over ocean at nadir incidence, we find for the 1 November 1985 dataset: $s\left(\lambda_{X}\right)=3.2 \mathrm{~dB}, s\left(\lambda_{K}\right)=3.4 \mathrm{~dB}$ and $\rho\left(\lambda_{X}, \lambda_{K}\right)$ $=0.81$, where notation $s(x)$ denotes the standard deviation of the random variable $x$. Therefore, the standard deviations for the SRT and DSRT attenuation estimates become

$$
s\left(A_{\mathrm{SRT}}\right)=[0.037+2.89]^{1 / 2}=1.7 \mathrm{~dB}
$$

and

$$
s\left(A_{\mathrm{DSRT}}\right)=[0.074+1.04]^{1 / 2}=1.06 \mathrm{~dB} .
$$

Assuming that the error incurred from the use of a $k-R$ relationship is small, then for a $5 \mathrm{~km}$ path length, the standard deviation in the rain rate $\left(\mathrm{mm} \mathrm{h}^{-1}\right)$ is approximately equal to the standard deviation in the path-attenuation $(\mathrm{dB})$. For example, an error of $1 \mathrm{~dB}$ in $A_{\mathrm{DSRT}}$ or $A_{\mathrm{SRT}}$ translates into a standard deviation in $R$ of about $1 \mathrm{~mm} \mathrm{~h}^{-1}$.

To compare the above values to the measured data, consider regressions of the form $R=a+b R_{X}$, where $R$ is the rain rate as found either from the SRT or DSRT and the independent variable, $R_{X}$, is taken from the $Z_{X}-R$ results. For the flight of 1 Nov., the rms deviation of the data from the regression line (denoted by the standard error of estimate, $\mathrm{SE}$ ) gives $\mathrm{SE}=2 \mathrm{~dB}$ when using the SRT as the dependent variable and SE $=1.1 \mathrm{~dB}$ for the DSRT as the dependent variable. These values agree reasonably well with the theoretical values of $1.7 \mathrm{~dB}$ and $1.06 \mathrm{~dB}$ computed above.

For the flight of 9 July 1986, the statistics of $\sigma^{\circ}$ in rain-free areas over the ocean are: $s\left(\lambda_{X}\right)=5.56 \mathrm{~dB}$, $s\left(\lambda_{K}\right)=4.1 \mathrm{~dB}, \rho\left(\lambda_{X}, \lambda_{K}\right)=0.95$. From Eqs. (15) and (17):

$$
\begin{aligned}
s\left(A_{\mathrm{SRT}}\right)=2.1 \mathrm{~dB} & (\mathrm{SE}=1.5 \mathrm{~dB}) \\
s\left(A_{\mathrm{DSRT}}\right)=1.1 \mathrm{~dB} & (\mathrm{SE}=0.9 \mathrm{~dB}) .
\end{aligned}
$$

Over land, $s\left(\lambda_{X}\right)=7.7 \mathrm{~dB}, s\left(\lambda_{K}\right)=6.3 \mathrm{~dB}, \rho\left(\lambda_{X}, \lambda_{K}\right)$ $=0.96$. Therefore,

$$
\begin{aligned}
s\left(A_{\mathrm{SRT}}\right)=3.2 \mathrm{~dB} & (\mathrm{SE}=2.4 \mathrm{~dB}) \\
s\left(A_{\mathrm{DSRT}}\right)=1.3 \mathrm{~dB} & (\mathrm{SE}=1.6 \mathrm{~dB}) .
\end{aligned}
$$

For purposes of comparison, the standard errors of estimate, as computed from procedure described above, are shown in parentheses. The results suggest that the DSRT is more accurate than the SRT as long as the correlation in $\sigma^{\circ}$ at the two wavelengths is sufficiently high. This appears to be the case not only for the 1 November and 9 July flights considered here but for five of the six datasets that have been analyzed. Although the correspondence between theory and experiment as indicated by the above results is only fair, the data do show that an approximation to the accuracy of the surface reference methods can be found from the rain-free statistics of $\sigma^{\circ}$ alone. It might be worth 
remarking that the accuracy of the SR methods over land might be improved by using as reference values the measurements over the same terrain when rain is absent. The reduction in the variance of the estimate will be proportional to temporal correlation in $\sigma^{\circ}$ between the rain and no-rain cases.

As shown in Tables 2-4, the SRT is positively biased with respect to the majority of rate estimates. Equation (14) implies that a positive bias will occur if the mean value of $\sigma^{\circ}$ outside the rain is larger than within the rain. Fujita et al. (1985) noted a similar bias in the SRT estimate and ascribed this to a roughening of the surface (and therefore a decrease in $\sigma^{\circ}$ ) caused by the rain drops striking the surface. As a check of this, data at off-nadir incidence have been processed; despite some inconsistencies, the general tendency is for the bias in the SRT to decrease as the incidence angle increases. This appears to be consistent with the above hypothesis in that roughening will tend to depress $\sigma^{\circ}$ at near-nadir angles and augment it for angles greater than about $10^{\circ}$ (Ulaby et al. 1982; Jones et al. 1977). A bias is introduced into the SRT if the presence of rain modifies the surface scattering characteristics. For the DSRT, however, even if such a change occurs, the bias will be small as long as the two wavelengths respond in a similar fashion [Eq. (16)]. To the extent that this is true, the bias in the DSRT will be smaller than in the SRT. Indeed, for the light rain rate examples discussed above, the mean values of the DSRT are in

TABLE 4. Statistics for 20 June 1986 (morning) flight $(N=2026)$.

a. Mean and standard deviation

\begin{tabular}{lcc} 
Method & $\begin{array}{c}\text { Mean } \\
\left(\mathrm{mm} \mathrm{h}^{-1}\right)\end{array}$ & $\begin{array}{c}\text { Std Dev } \\
\left(\mathrm{mm} \mathrm{h}^{-1}\right)\end{array}$ \\
\hline$Z_{X}-R$ & 3.8 & 2.8 \\
$Z_{K}-R$ & 2.1 & 0.88 \\
DSRT & 4.7 & 4.1 \\
SRT & 5.8 & 4.0 \\
DWT & 5.1 & 4.3 \\
DWAV & 4.7 & 4.2
\end{tabular}

b. Matrix of correlation coefficients

\begin{tabular}{lccccc} 
& $Z_{K}-R$ & DSRT & SRT & DWT & DWAV \\
\hline$Z_{X}-R$ & \multirow{2}{*}{0.72} & 0.84 & 0.80 & 0.66 & 0.64 \\
$Z_{K}-R$ & & 0.56 & 0.54 & 0.51 & 0.47 \\
DSRT & & & 0.94 & 0.77 & 0.78 \\
SRT & & & & 0.76 & 0.75 \\
DWT & & & & & 0.95
\end{tabular}

c. Normalized error

\begin{tabular}{llllll}
$Z_{X}-R$ & 0.94 & 0.58 & 0.65 & 0.77 & 0.79 \\
$Z_{K}-R$ & & 1.30 & 1.30 & 1.35 & 1.37 \\
DSRT & & & 0.35 & 0.59 & 0.60 \\
SRT & & & & 0.53 & 0.60 \\
DWT & & & & & 0.30 \\
\hline
\end{tabular}

better agreement with the $Z_{X}-R$ method than is the SRT.

Unfortunately, other sources of bias exist. The surface reference methods estimate the total path attenuation which can include contributions both from the rain and nonliquid hydrometeors. By assuming a single $k-R$ law for rain along the entire path, a bias will be introduced which can be either negative or positive, depending on the proportion of snow, ice and brightband. Effects of cloud liquid water will enhance the measured attenuation and lead to overestimates in the rain rate (Joss et al. 1974). One other source of bias will be caused by reflectivity gradients within the beam. As an extreme example of this, consider the antenna beam to be half filled with rain. Since the measurement of the surface return can be no smaller than $3 \mathrm{~dB}$ below the value which would be measured in the absence of rain, the estimates will be negatively biased. Although these bias terms can be significant, it is important to note that they will affect the SRT and DSRT estimates in similar ways. When the mean values of the SRT and DSRT differ (as they do in the above examples) the most likely reason for the discrepancy are changes in the average values of $\sigma^{\circ}$ within and outside the rain.

The main sources of error in the dual-wavelength method are: finite sampling (Srivastava and Carbone 1971), occurrences of non-Rayleigh scattering (Eccles and Mueller 1971; Nakamura et al. 1984) which invalidate the assumption that $Z$ is wavelength independent, low signal to noise ratios caused by excessive attenuation at the shorter wavelength, mismatched beams (Rinehart and Tuttle 1982), and reflectivity gradients within the beam.

To assess the contribution to the total error from each of these sources is quite difficult. A few qualitative statements, however, can be made.

1) The interval over which the method is applied should not include range gates where significant Mie scattering occurs or where the surface or noise contributions are comparable to the rain return.

2) Some of the effects of finite sampling can be inferred by comparisons between the DWT and DWAV (e.g., Fig. $4 a$ and $4 b$ ). The decrease in the magnitude of fluctuations that can be seen by comparing Fig. $4 \mathrm{~b}$ with Fig. 4a can be attributed primarily to the use of a greater number of independent samples. The large residual variance, however, appears to imply that other more significant sources of error remain.

3) Although the results of Figs. 3a and 5a imply that the correlation between the reflectivity factors at the two wavelengths is excellent at low rain rates, it would be incorrect to conclude that this high degree of correlation continues to hold at higher rain rates since the effects of non-Rayleigh scattering will generally increase with rain rate. On theoretical grounds, the effects of non-Rayleigh scattering would seem to contribute the largest amount of error. 
Although the $Z_{X}-R$ method is used throughout the paper as a standard of comparison, the errors in the method have been well documented (Zawadzki 1984). Some of the most important are: biases caused by errors in the radar constant, reflectivity gradients, and attenuation effects at the high rain rates. One of the primary errors arises from the use of a $Z-R$ relationship that assumes a distribution of drop sizes and drop velocities which differ from the actual distributions. Because no measurements of the DSD were made during these flights, the effects of $Z-R$ fluctuations and biases are difficult to assess. Moreover, a light rain rates where the attenuation methods are generally less accurate than a well-calibrated radar using the $Z-R$ method, comparisons between the $Z-R$ and attenuation methods are not particularly helpful in identifying the errors in the $Z-R$ method. For cases of moderate and high rain rates, the situation changes and at least some qualitative comments can be made.

\section{c. Moderate rain rate cases}

Temporal plots of the path-averaged rainfall rate from the SRT, DSRT and $Z_{X}-R$ are presented in Fig. 6 for a flight on 12 June 1986. Nearly all the data were measured over a land background. Using as before the three central nadir incidence angles, we obtain correlation coefficients for the DSRT and $Z_{X}-R$ of 0.65 , for the SRT and $Z_{X}-R$ of 0.76 and for the SRT and DSRT of 0.82 . In each case the number of observations, $N$, is equal to 961 . These coefficients might appear somewhat lower than would be expected from a visual inspection of the plots. In fact, the primary reason for this is due to poor correlations between the methods at high and very low rain rates. In particular, at the higher rain rates, the surface reference methods often yield lower values of rain rate than the $Z_{X}-R$. Examination of the curves of the return power versus range for these time periods shows that these occurrences sometimes correspond to the loss of the $K_{a}$-band surface return; that is, because of large $K_{a}$-band attenuation, the surface return is indistinguishable from the system noise. Although the surface return is not apparent at $K_{a}$-band, the location of the surface can be inferred from the $X$-band return. The measured power consists of the system noise $N$ and the surface return $S$. By using $N+S$ as an estimator of $S$, the surface return within the rain is overestimated, thereby producing underestimates in the rainfall rate. Another possible cause of the differences are reflectivity gradients across the beam which result in underestimates in the surface reference methods and overestimates in the $Z-R$ method. This source of error may have been significant for this flight where a number of isolated convective cells were encountered.

By eliminating cases of high and very low rain rates, the correlations improve. For example, by selecting only those results for which the rain rates are between $0.1 \mathrm{~mm} \mathrm{~h}^{-1}$ and $25 \mathrm{~mm} \mathrm{~h}^{-1}$, the correlation coefficient for the DSRT and $Z_{X}-R$ is $0.78(N=852)$ and for the SRT and $Z_{X}-R$ is $0.87(N=785)$ with a correlation coefficient for SRT and DSRT of $0.98(N=748)$. These results point to an obvious but important feature of attenuation methods: They perform best in the intermediate range of rain rates where the attenuation is large enough to be detectable but not so severe as to cause a loss of signal.

By moving to a dataset collected over an ocean background, the dynamic range for the surface reference methods improves because of an increase in $\sigma^{\circ}$. Using the same format as above, we plot in Fig. 7 the rain rates derived from data measured at a near-nadir incidence angle over ocean on the morning of 20 June 1986. As in Figs. 3 and 4 , the $Z_{X}-R$ estimates are represented by the dotted curves while the DSRT and DWAV are represented by the solid lines in $7 \mathrm{a}$ and $7 \mathrm{~b}$, respectively. At moderate to high rain rates, the correlation between the DSRT and $Z_{X}-R$ methods is good (Fig. 7a) but the magnitude of the DSRT results are significantly larger.

To assist in the interpretation of these differences, we consider the corresponding results from the dualwavelength estimate, DWAV (Fig. 7b). Focusing on the region from 450 to 520 , we find that the attenuation methods give consistently larger rainfall rates than those obtained from the $Z_{X}-R$ method. Examining the plots of radar return power versus range during this period shows that the aircraft was at a $3 \mathrm{~km}$ altitude, just below the bright band. (The presence of a bright band, and its location with respect to the aircraft can be obtained from an inspection of the mirror-image return.) In addition, the rain reflectivity at $X$-band is fairly uniform with range and both $K_{a}$-band and $X$-band signals are above the system noise level. As the dual-wavelength and SR methods provide virtually independent measurements of attenuation and as the path is below the bright band, the probable reasons for the discrepancies between the $Z_{X}-R$ and attenuation methods can be narrowed to: 1 ) attenuation effects at $X$-band, 2) errors in the radar constants, and 3 ) deviations from the assumed drop size distribution (DSD) leading to errors in the $k-R$ and $Z-R$ laws.

To check the first possibility, we use for the attenuation coefficient at $X$-band the relationship: $k$ $=0.011 R^{1.2}$. For a rain rate of $15 \mathrm{~mm} \mathrm{~h}^{-1}$, the total two-way attenuation is $2 \mathrm{kr}$, which with $r=3 \mathrm{~km}$, equals $1.7 \mathrm{~dB}$. This implies that on average the corrected reflectivity would be $22 \%$ larger than the measured value, which translates into a corrected rain rate (using $Z_{X}=a R^{1.5}$ ) $14 \%$ larger. Although this serves to reduce the differences, it accounts for only a part of the discrepancy.

Regarding the second possibility, and assuming a 3 $\mathrm{dB}$ error in the $X$-band radar constant, then the use of 
a $Z_{X}-R$ relationship of the form $Z_{X}=a R^{1.5}$ yields rainfall rates that are $63 \%$ as large as the correct value. The dual-wavelength methods, on the other hand, are independent of such errors as long as the radar constants do not change during the course of a single observation $(1 / 3 \mathrm{~s})$. For the surface reference methods, the situation is more complicated because the errors depend on the differences in the radar constants between periods of rain and no-rain.

One other possible explanation for the observed differences is the variation in the DSD. Atlas and Ulbrich (1977) and Ulbrich (1983) have shown that for wavelengths near $0.9 \mathrm{~cm}$ the $k-R$ relationship is much less dependent on the DSD than is the $Z-R$ law near $3 \mathrm{~cm}$. It is consistent with these studies that the $Z-R$ relationship that we have used may be inaccurate for this time period even though the $k-R$ law is basically correct. As an example, if the $Z_{X}-R$ relationship, $Z_{X}$ $=200 R^{1.5}$ were employed rather than the one in use $\left(Z_{X}=300 R^{1.5}\right)$, the rain rate estimates would be increased by $31 \%$.

A feature of Fig. 7 more easily explainable is the large values of rain rate estimated by the DWT and DWAV methods that occur between observation numbers 200 and 250. Examination of the corresponding return power plots shows that the $X$-band reflectivities peak approximately $1 \mathrm{~km}$ away from the aircraft and decrease rapidly near the surface. The $K_{a^{-}}$ band return, moreover, is noise dominated over the last kilometer of the rain. Because of the manner in which the intervals for the DWT and DWAV algorithms are selected, the rain rate is computed only over the top portion of the storm where the rain rate is most intense. More generally, it should be noted that the three methods have different regions of application. For example, in Fig. 1b, the $Z_{X}-R$ and dual-wavelength methods are applied over the intervals $A^{\prime} C$ and $A^{\prime} C^{\prime}$, respectively, while the SR estimates are proportional to the attenuation over the entire path. Therefore, in cases of nonuniform precipitation, the discrepancies in the rain rates can be ascribed in part to differences in the interval of application.

A summary of results for this flight is given in Table 4. Shown first are the mean rain rates for the 2320 observations made at near-nadir incidence. The attenuation methods typically yield mean rain rates between about 1 to $2 \mathrm{~mm} \mathrm{~h}^{-1}$ larger than those estimated by the $Z_{X}-R$. As in the light rain rate cases, the SRT gives the largest mean value while the $Z_{K}-R$ method gives the smallest. With respect to the correlation coefficient and normalized error, the surface reference methods are in somewhat better agreement with the $Z_{X}-R$ than either the DWT or DWAV. Scatter plots of the DSRT versus $Z_{X}-R$ and the DWAV versus $Z_{X}-R$ are shown in Figs. 8a and $8 \mathrm{~b}$, respectively. The data points were obtained at near nadir incidence $\left(<5^{\circ}\right)$. For purposes of comparison, the same pairs of quantities are plotted in Figs. $9 \mathrm{a}, 9 \mathrm{~b}$ for cases of off-nadir incidence $\left(5^{\circ}-\right.$ $20^{\circ}$ ). The results show that in going from near- to offnadir incidence the correlation coefficient for DSRT and $Z_{X}-R$ decreases from 0.84 to 0.82 while that for the DWAV and $Z_{X}-R$ increases from 0.64 to 0.72 .

For the sake of completeness, we show in Table 5 the matrix of correlation coefficients for the two remaining data flights which were performed on 20 June 1986 (afternoon) and on 2 Oct. 1985. In general, good correlations are obtained for the attenuation methods and the $Z_{X}-R$. It should be noted that the correlation coefficients for the $Z_{X}-R$ and the various attenuation methods for the 2 Oct. flight were the highest of the six analyzed. On the other hand, the mean values of the $Z_{X}-R$ derived rain rates were found to be about a factor of 2 smaller than those obtained from the attenuation methods. The most likely reasons for the discrepancies are errors in the radar constant or an incorrect choice of the $Z_{X}-R$ relationship.
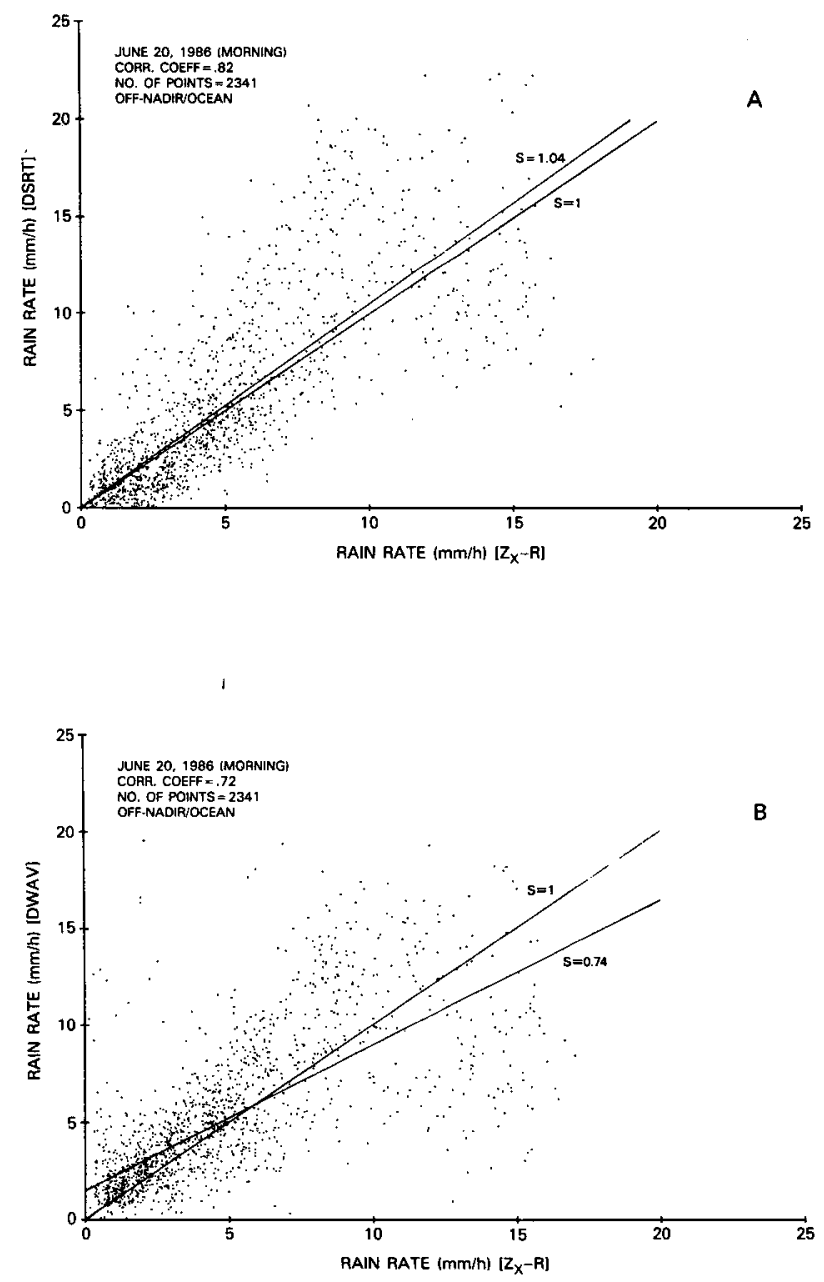

FIG. 9. Same as Fig. 8 but for off-nadir incidence angles between $5^{\circ}$ and $20^{\circ}$. 
TABLE 5. Matrix of correlation coefficients

a. 20 June 1986 (afternoon) $(N=1454)$

\begin{tabular}{lccccc} 
& $Z_{K}-R$ & DSRT & SRT & DWT & DWAV \\
\hline$Z_{X}-R$ & 0.77 & 0.86 & 0.86 & 0.84 & 0.71 \\
$Z_{K}-R$ & & 0.72 & 0.74 & 0.78 & 0.76 \\
DSRT & & & 0.95 & 0.84 & 0.71 \\
SRT & & & & 0.85 & 0.72 \\
DWT & & & & & 0.78
\end{tabular}

b. 2 Oct. $1985(N=2623)$

\begin{tabular}{llllll}
$Z_{X}-R$ & 0.65 & 0.91 & 0.91 & 0.9 & 0.85 \\
$Z_{K}-R$ & & 0.49 & 0.47 & 0.58 & 0.53 \\
DSRT & & & 0.98 & 0.8 & 0.74 \\
SRT & & & & 0.81 & 0.75 \\
DWT & & & & & 0.90 \\
\hline
\end{tabular}

\section{Summary and conclusions}

For most of the proposed designs for spaceborne meteorological radars, frequencies have been chosen at $10 \mathrm{GHz}$ and above (Thiele 1987; Barrett and Martin 1981). Among the reasons for this selection is the desire to attain adequate spatial resolution. Apart from the usual errors in the $Z-R$ (reflectivity factor-rainfall rate) method, the error caused by attenuation can be significant. Methods which use the attenuation to infer rainfall rate offer the potential benefits of increased dynamic range, independent estimates of the precipitation parameters, and the avoidance of some of the errors inherent in the $Z-R$ method.

In this paper several methods were applied to measurements made by an airborne dual-wavelength radar operating at $0.87 \mathrm{~cm}\left(K_{a}\right.$-band $)$ and at $3 \mathrm{~cm}(X$-band $)$ for the purpose of estimating path-averaged rainfall rates. These methods include the traditional $Z-R$ methods (designated by $Z_{X}-R$ and $Z_{K}-R$ for the $X$ and $K_{a}$-band wavelengths, respectively), single- and dual-wavelength surface reference techniques (SRT and DSRT, respectively), and standard dual-wavelength methods with and without range-averaging (DWT and DWAV, respectively).

Correlation coefficients between the rainfall rates found using the $Z_{X}-R$ and DSRT methods are generally between 0.7 and 0.9 where the best correspondence usually occurs in stratiform rain over an ocean background at near-nadir incidence. Although most of the measurements were made over ocean, the results over land indicate that the dynamic range of the surface reference methods decreases (because of the reduction in $\sigma^{\circ}$ ) and the variance increases relative to an ocean background. Comparisons between the single and dualwavelength surface reference techniques suggest that the latter is usually preferable. Two virtues of the DSRT, which the SRT does not share, are that the variance decreases as the wavelength correlation of $\sigma^{\circ}$ increases and that the bias remains small even if $\sigma^{\circ}$ changes between raining and rain-free areas. Some reduction in the variance of both methods might be possible over land by using measurements of the surface return at the actual location of the rain measurement before or after the occurrence of rain. Such measurements, however, have not been performed.

Correlation coefficients between the $Z_{X}-R$ and the standard dual-wavelength methods were found to be somewhat less than that between the DSRT and $Z_{X^{-}}$ $R$. The correlations are further degraded if no attempt is made to eliminate regions of strong non-Rayleigh scattering such as the melting layer. In this paper, a simple method is used to detect such regions; improved algorithms or interactive processing, where the brightband is often visually obvious, should further reduce the variance.

Two variations of the dual-wavelength method were considered: one with and one without range-averaging. Contrary to expectations, range averaging does not consistently reduce the variance and, in fact, often produces poorer correlations with the $Z_{X}-R$ method than the estimate using no averaging. Although the reasons for the lack of improvement are not well understood, one possibility is that the nonuniform averaging biases the estimate toward the rain rates at the center of the interval. Tests of range-averaging methods need to be conducted as a function of the nonuniformity of the reflectivity over the interval.

Because the primary sources of error for the three methods ( $Z-R$, dual-wavelength and surface reference) are virtually independent, when the rain rate estimates agree, some confidence in the results is warranted. Cases of good agreement occur most often in stratiform rain for rain rates from a few $\mathrm{mm} \mathrm{h}^{-1}$ to about $15 \mathrm{~mm}$ $\mathrm{h}^{-1}$; that is, for cases where the rain is clearly distinguishable from other types of precipitation and where the attenuation is detectable at the shorter wavelength but not so severe as to cause a loss of signal. When the methods are not in agreement, the disparity sometimes can be explained by examining plots of the radar return power and from a knowledge of the major error sources. Some of the obvious sources of difference are: Loss of the $K_{a}$-band rain or surface return leading to biases in the dual-wavelength and surface reference methods; differences in the interval of application in cases of nonuniform reflectivity; and cases of light or heavy rain rates where the attenuation methods and the $Z$ $R$ method, respectively, are subject to large errors.

Although it seems clear that the $Z-R$ method should be used in cases of light rain (where attenuation at the shorter wavelength is negligible) and an attenuation method at high rain rates (where attenuation is significant at the longer wavelength), in the intermediate range of rain rates, the "best" estimate is far from obvious. When two out of the three methods are in agreement, a reasonable procedure would be to try to explain the source of error in the third. For example, when the 
attenuation estimates differ from the $Z-R$, the assumed drop size distribution might be incorrect. This assumption might be tested by examining the rain type, the location of the measurement within the storm, the areal extent of the rain, etc., to determine whether a different DSD is reasonable. In cases of uniform reflectivity in range, methods that estimate a two-parameter DSD should be useful (Goldhirsh and Katz 1974; Ulbrich and Atlas 1978). Similarly, if the surface reference estimates differ from the others, the likely causes are reflectivity gradients across the beam, contributions from hydrometeors in and above the melting layer or sigma zero anomalies: information concerning the first two can be obtained from plots of the return powers versus range, while insight into the surface scattering may be possible by the comparison of different surface reference measurements or from the mirror-image return.

Further testing and refinements of these and other attenuation methods are required not only for the estimation of path-average rain rate but for their capabilities in range profiling, in the discrimination of various types of precipitation (hail, snow, rain), and in estimating other precipitation parameters. The success or failure of such methods affect not only the methods available for the analysis of airborne and spaceborne radar data but determine, in part, the basic design philosophy.

\section{REFERENCES}

Atlas, D., 1954: The estimation of cloud parameters by radar. $J$. Meteor., 11, 309-317.

measurement by microwave attenuation in the $1-3 \mathrm{~cm}$ band. J. Appl. Meteor., 16, 1322-1331.

Barrett, E. C., and D. W. Martin, 1981: The Use of Satellite Data in Rainfall Monitoring. Academic Press, 240 pp.

Berjulev, G. P., and V. V. Kostarev, 1974: Dual-wavelength radar measurements of $8 \mathrm{~mm}$ radiowave attenuation by atmospheric attenuation and clouds. J. Rech. Atmos., 8, 358-363.

Eccles, P. J., 1979: Comparison of remote measurements by singleand dual-wavelength meteorological radars. IEEE Trans. Geosci. Electron., 17, 205-218.

$\longrightarrow$, and E. A. Mueller, 1971: $X$-band attenuation and liquid water content estimation by a dual-wavelength radar. J. Appl. Meteor., 10, 1252-1259.

Fujita, M., K. Okamoto, S. Yoshikado and K. Nakamura, 1985: Inference of rain rate profile and path-integrated rain rate by an airborne microwave rain scatterometer. Radio Sci., 20, 631642.
Goldhirsh, J., and I. Katz, 1974: Estimation of raindrop size distribution using multiple wavelength radar systems. Radio Sci., 9, 439-446.

Jones, W. L., L. C. Schroeder and J. L. Mitchell, 1977: Aircraft measurements of the microwave scattering signature of the ocean. IEEE Trans. Antennas Propag., 25, 52-61.

Joss, J., R. Cavalli and R. K. Crane, 1974: Good agreement between theory and experiment for attenuation data. J. Rech. Atmos., 8, 299-318.

Kostarev, V. V., and A. A. Chernikov, 1968: The adjustment of radar estimates of rainfall with radar attenuation data. Preprints, 13th AMS Conf. on Radar Meteorology, Boston, Amer. Meteor. Soc., 396-399.

Meneghini, R., 1977: Spatially averaged estimates of attenuation using dual frequency radar techniques. NASA X-953-77-71. (Available from author or the National Technical Information Service, Springfield, VA 22161.)

- , and D. Atlas, 1986: Simultaneous ocean cross section and rainfall measurements from space with a nadir-looking radar. J. Atmos. Oceanic Technol., 3, 400-413.

- J. Eckerman and D. Atlas, 1983: Determination of rain rate from a spaceborne radar using measurements of total attenuation. IEEE Trans. Geosci. and Remote Sensing, 21, 34-43.

- J. A. Jones and L. H. Gesell, 1987: Analysis of a dual-wavelength surface reference radar technique. IEEE Trans. Geosci. and Remote Sensing, 25, 456-471.

Moore, R. K., 1981: Use of combined radar and radiometer systems in space for precipitation measurement-some ideas. NASA Workshop Report: Precipitation Measurements from Space. D301-D325. (Available from the National Technical Information Service, Springfield, VA 22161.)

Nakamura, K., H. Inomata and J. Awaka, 1984: Influence of raindrop distributions on $X$ - and $K_{a}$-band dual-wavelength radar. Preprints, 22nd Conf. on Radar Meteorology, Boston, Amer. Meteor. Soc., 400-404

Okamoto, K., S. Yoshikado, H. Masuko, T. Ojima, N. Fugono, K. Nakamura, J. Awaka and H. Inomata, 1982: Airborne microwave rain-scatterometer/radiometer. Int. J. Remote Sens., 3, 277-294.

Rinehart, R. E., and J. D. Tuttle, 1982: Dual-wavelength processingthe effects of mismatched antenna beam patterns. Proc. URSI Commission F, Multiple Parameter Measurements of Precipitation, Bournemouth, U.K., 83-88.

Srivastava, R. C., and R. E. Carbone, 1971: The effect of signal fluctuation on the performance of dual-wavelength radar hail-detector. Lab. for Atmos. Probing, Univ. of Chicago, Tech. Rep. $20,25 \mathrm{pp}$.

Thiele, O. W., Ed., 1987: On the requirements for a satellite mission to measure tropical rainfall. NASA Reference Publication 1183, 72 pp.

Ulaby, F. T., R. K. Moore and A. K. Fung, 1982: Microwave Remote Sensing, Vol. 2. Addison-Wesley, 1064 pp.

Ulbrich, C. W., 1983: Natural variations in the analytical form of the raindrop size distribution. J. Climate Appl. Meteor., 22, 1764-1775.

- and D. Atlas, 1978: The rain parameter diagram: Methods and applications. J. Geophys. Res., 83, 1319-1325.

Zawadzki, I., 1984: Factors affecting the precision of radar measurements of rain. Preprints, 22nd Conf. on Radar Meteorology, Boston, Amer. Meteor. Soc., 251-256. 